University of Nebraska - Lincoln

DigitalCommons@University of Nebraska - Lincoln

2019

\title{
A Mobile Solution to Drug Prescription and Dispensation with Privacy Protection for Developing Economies
}

Kingsley C. Nwosu

Follow this and additional works at: https://digitalcommons.unl.edu/jade

Part of the Econometrics Commons, Growth and Development Commons, International Economics Commons, Political Economy Commons, Public Economics Commons, and the Regional Economics Commons

This Article is brought to you for free and open access by the Institute for the Advancement of Developing Economies at DigitalCommons@University of Nebraska - Lincoln. It has been accepted for inclusion in Journal for the Advancement of Developing Economies by an authorized administrator of DigitalCommons@University of Nebraska - Lincoln. 


\title{
A Mobile Solution to Drug Prescription and Dispensation with Privacy Protection for Developing Economies
}

\author{
Kingsley C. Nwosu* \\ Saint Leo University, Newport News, Virginia, USA
}

\begin{abstract}
It is unarguable that many countries within the developing economies have not been able to reap many of the benefits from the innovations in Computer and Information Technologies (CIT) as they pertain to the advances in healthcare delivery systems. The contributing factors to the problems in these countries are multifaceted, such as, ethnic, cultural, environmental, governance, and infrastructural. To address these problems, current efforts have focused mostly on the issues of disease control and management. However, insufficient attention has been given to the concomitant problems of medication (drug / prescription) management especially as they relate to information management between patients, physicians, and pharmacists. In most of these countries, the absence of integrated and reliable system for management and maintenance of a patient's medical history exposes a patient to prescription and dispensation errors and/or mistakes by physicians and pharmacists, respectively. In this paper, taking cognizance of the infrastructural and other applicable impediments and deficiencies, the design and implementation of a mobilebased system (henceforth referred to as "iRx") is presented. This system enables patients, physicians, and pharmacists to reliably interact in managing and maintaining an individual 's drug history. This management is subject to the patient's authorization for anyone to access the applicable information.
\end{abstract}

Keywords: Developing economies, drugs/prescriptions delivery, healthcare delivery, mobile technology, under-developed economies, mHealth.

*Corresponding author: kingsley.nwosu@saintleo.edu

\section{INTRODUCTION AND LITERATURE REVIEW}

Many factors are attributable to the deplorable conditions of healthcare delivery in many developing countries. According to Ameh (2005), many of the contributing factors are ethnic and cultural; and exacerbated by poverty, ignorance, lack of resources and health facilities, and bad leadership. Many of the factors, issues, consequences, disparities, and solutions in healthcare delivery in developing economies are discussed in da Cruz et al. (2017); WHO (2005); Robertson, DeHart, Tolle, and Heckerman (2015); and Peters et al. (2008). Based on the findings of Tam et al. (2005) on the frequency of medical errors in medication prescription, the authors (da Cruz et al., 2017) presented the development of a medication history from the medical records to measure the factors associated with medication errors and concluded that medication errors are known to compromise patient safety.

In Mills (2014), the author finds that increasing attention has been paid to the importance of healthcare systems in recent years. However, in low- and middle-income countries (LMICs), 
substantial external funding has been provided for disease-specific programs without commensurate attention to the issues of the broader healthcare infrastructures. In Peters et al. (2011), the authors synthesized a compendium of strategies to improve health services delivery in LMICs and analyzed the findings. They provided some suggestions for what works and how to improve implementation. However, the authors argued that not nearly enough attention has been paid to demonstrating how to improve services for the poor. They concluded that approaches that use information intelligently are necessary for successful implementation of health services.

According to Epstein and Bing (2011), "A child in a developing country is 33 times as likely to die before age five as a child in the industrialized world, and up to 60 percent of these deaths could be prevented through basic health education, products, and services ...". The authors argued that developing countries lack delivery systems that can give their populations access to health information, health technologies, and services, and to high-quality and affordable medications. After analyzing the pros and cons of the healthcare delivery channels in developing economies, the authors projected that cell phones, computers, and the Internet are likely to revolutionize the treatment and prevention of disease as they become more accessible to the developing world.

Addressing the critical challenge of healthcare access in LMICs, Bangdiwala, Fonn, Okoye, and Tollman (2010) looked at the issue of massive global shortage of workforce resources. They postulated that a strong workforce is essential, therefore, building the human resources for health should encompass community health workers, mid-level workers and strengthened primary healthcare systems. In furthering the consequences of lack of access to health technologies in developing countries, Frost and Reich (2009) argued that although the issue of access to medicines and other technologies has risen on the global policy agenda, the focus on certain types of access barriers (especially pricing and patents) has tended to obscure other important obstacles to access such as distribution, delivery, and adoption problems.

Worldwide shortage of health professionals is more pronounced in rural areas of developing countries; and rural and remote inhabitants experience lower life expectancy and poorer health status according to Strasser, Kam, and Regaladol (2016). The authors concluded that greater health equity can be achieved via their conceptual framework of access to primary health care, sustainable rural health service models, rural health workforce supply, and policy implications.

Using the increasing cost of healthcare in developed and developing economies, with the concomitant effects on healthcare delivery, Anyika (2014) explored the relationship between environmental uncertainty and health care delivery system in Nigeria. The study was based on reviewing the dynamics of health care delivery in some developed economies and Nigeria with regards to methods of adaptation.

Other researchers (including American Public Health Association, 2008) have concluded that many people in low income countries do not practice rudimentary disease prevention measures such as keeping water covered, washing vegetables, brushing teeth, vaccinating children, taking the garbage away from the house and screening windows against flies and mosquitoes. Hospitals and clinics sometimes have good medicines and vaccines but lack consistent and reliable infrastructure such as refrigerators, clean syringes and nurses--- to keep them safe and adequately deliver them. Sometimes needles are reused, spreading disease and infection; and vaccines are given though their 
effectiveness has been compromised by lack of proper preservation (refrigeration). In those communities, much of the health care duties are taken care of by village midwives, who not only help deliver children but also perform check-ups, give health care advice, dispense birth control devices, and provide post-natal care.

Broadly speaking, currently available types of mobile health (mHealth - part of the wider "eHealth" domain) technologies for patients and healthcare providers are grouped into (a) Mobile health apps providers, (b) Mobile health apps for consumers (c) Patient portals, (d) Mobile-enabled EHRs (e) Secure text messaging (STM), (f) Patient monitoring devices, and (g) Telemedicine. These applications help in clinical decision support during patient visits; patient monitoring, tracking and follow-up, fitness tracking, facilitating provider/staff and patient communication for test results, prescription refills, medical record review; ubiquitous access to Electronic Health Records (EHRs) by providers; securing exchange of messages between devices and office workstations; remote health monitoring and improve treatment compliance by patients; and real-time review of lab results, seeing patients, prescribing treatment via cloud-based health information technology.

However, for developing countries healthcare providers mainly use mobile healthcare technologies for education and awareness, remote data collection, remote monitoring, communication and training for healthcare workers, disease and epidemic outbreak tracking, and diagnostic and treatment support. In all cases, there is no single platform for the management and utilization of patient medical records by stakeholders. In developed countries, different network of healthcare providers provides their own healthcare applications; however, there are reliable means for enforcing patient privacy which are lacking in most of the developing countries.

Besides the environmental contributory factors to problems in healthcare in developing countries such as poor water quality, availability, and sanitation; vector-borne diseases; poor ambient and indoor air quality; toxic substances; and global environmental change, which are mainly addressable through government efforts and education, healthcare is still largely technologically limited. The technological solutions in developing countries, as reviewed above, have not explored the possibilities of deploying full-scale, mobile-based healthcare delivery system. Achieving this objective requires addressing one of the major components: mobile-based prescription drug management - which hitherto has received minor attention. In this paper, we explore the viability of designing, developing, and deploying an integrable mobile-based prescription management system that encompasses all the major healthcare stakeholders (Patients, Physicians, and Pharmacists); and reliably enforces patients' privacy.

\section{RELEVANT BACKGROUND}

The United Nations International Standard Industrial Classification (ISIC) categorizes the healthcare industry as generally consisting of:

1. Hospital activities;

2. Medical and dental practice activities;

3. Other human health activities, which involves activities of, or under the supervision of, nurses, midwives, physiotherapists, scientific or diagnostic laboratories, pathology clinics, residential health facilities, or other allied health professions (United Nations, 2008). 
In general, healthcare is the maintenance or improvement of health via the prevention, diagnosis, and treatment of disease, illness, injury, and other physical and mental impairments in human beings. A healthcare provider is an institution (such as a hospital or clinic) or person (such as a physician, nurse, allied health professional or community health worker) that provides preventive, curative, promotional, rehabilitative or palliative care services in a systematic way to individuals, families or communities.

Healthcare delivery is the most conspicuous and frequent activity in the healthcare system spectrum. It encompasses primary, secondary and tertiary levels of care where primary care is the day-to-day healthcare given by a healthcare provider. Secondary care is the necessary treatment for a short period of time for a brief but serious illness, injury, or other health condition usually rendered in an Emergency Room (ER). Tertiary care is specialized consultative healthcare, usually for in-patients and on-referral from a primary or secondary health professional, in a facility that has personnel and facilities for advanced medical investigation and treatment.

Healthcare delivery involves interactions between patients, medical professionals (physicians), and pharmacists. Patients present themselves to physicians for medical diagnoses and treatment, which usually involves prescribing medications for the patients; the medications are dispensed by the pharmacists. The interactions between the patients, physicians, and pharmacists with respect to medications require certain level of efficacy and privacy issues. Physicians need access to current and historical medication information from a patient; and they also need effective and reliable means of communication with the pharmacists. In most of the developed economies today, the prevalence of Information Technology (IT) infrastructure makes it possible and easy for physicians to have automated access to a patient's medication history; and to transmit required medication to specified pharmacists, thereby reducing the propensity for errors and mistakes. Unfortunately, in many developing economies, due to the absence, instability or inadequacies of the necessary IT infrastructure, there are no automated real-time means of accessing a patient's medication history or transmission of required medications, especially, when a patient visits a non-primary care physician. These situations pose grave consequences when a patient is uncommunicative or forgets a critical current medication.

\section{ABOUT iRx}

iRx is a mobile-based system that allows patients, physicians, and pharmacists to interact in managing and maintaining a patient's drug prescription history and other associated medical history information under the authorization and control of the patient. When an iRx patient visits an iRx physician, the physician can use the patient's iRx, subject to the patient's authorization, to review the patient's current medications. The physician can also, subject to the patient's authorization, update the patient's medication history on the patient's iRx device.

When an iRx patient visits a pharmacist, the pharmacist can also use the patient's iRx, subject to the patient's authorization, to review the patient's current medications; and update the patient's medications accordingly.

An iRx patient will be able to manage his/her medication history (and those of associated dependents) for those medications that he/she obtained without the assistance of either a physician 
or pharmacist. Besides the usual review by a physician or pharmacist, iRx is equipped with internal knowledge for drug interaction verification.

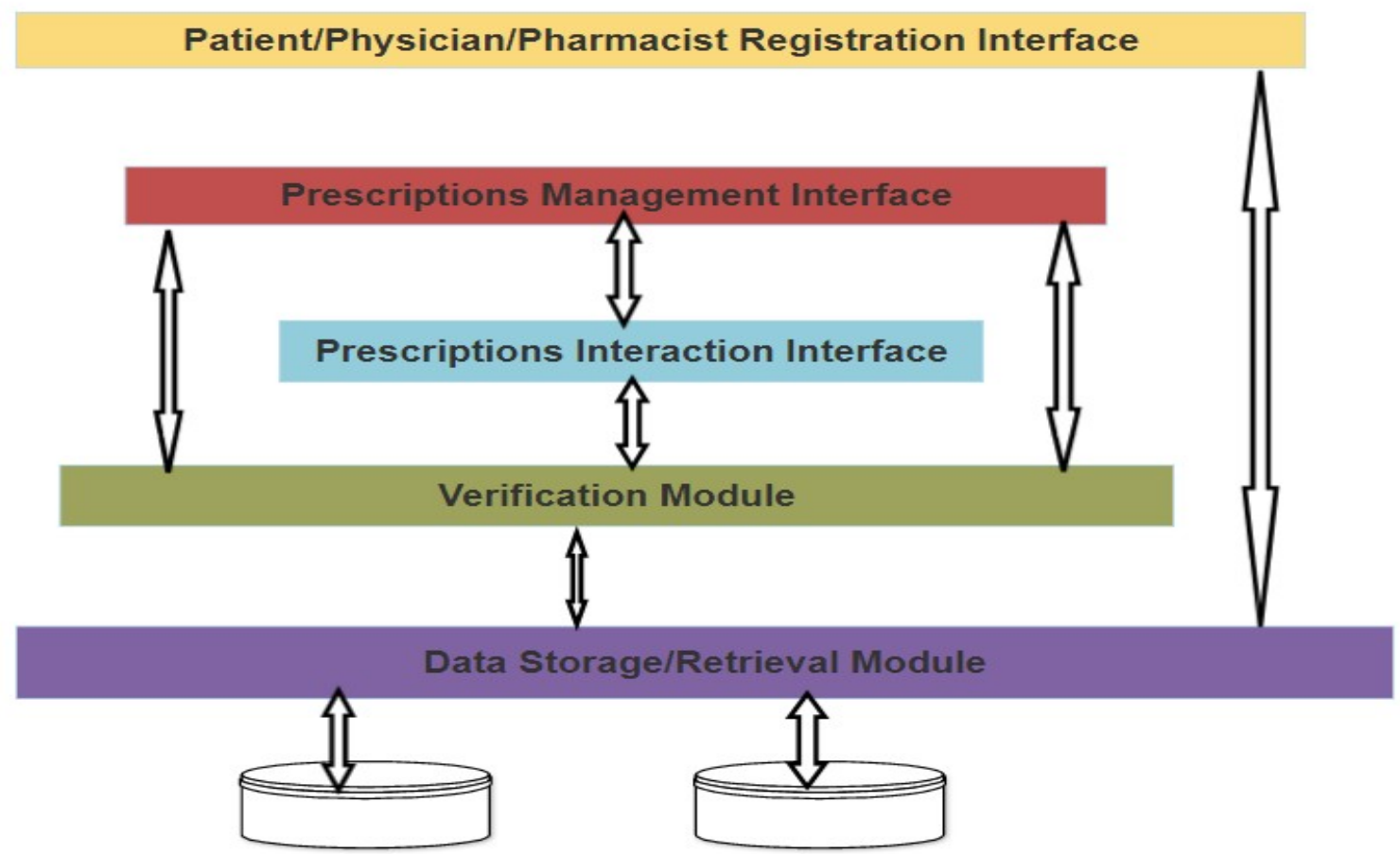

\section{Figure 1: iRx Architectural Interfaces}

\subsection{General Description of $\mathrm{iRx}$}

The iRx interface architecture (Fig. 1) is primarily made up of the following:

- Registration and Personal Interface (RPI),

- Prescriptions Management Interface (PMI),

- Prescriptions Interaction Interface (PII),

- Patient Verification Interface (PVI).

The Registration and Personal Interface (RPI) is used to register new iRx members and to update applicable personal information. During the registration, certain Personal Identifiable Data (PID) are collected. This interface is also required for the physicians' and pharmacists' registration.

The Prescriptions Management Interface (PMI) is used for the management of a patient's medications. It is used to maintain the history of active and inactive medications. This interface is accessible to the patients, physicians, and pharmacists.

The Prescriptions Interaction Interface (PII) is used by the patents, physicians, and pharmacists to quickly check for drug interaction of a given medication with existing active medications.

The Patient Verification Interface (PVI) is used by the physicians and pharmacists to reliably verify the identity of a patient. The verification module is also used before any updates by a physician or pharmacist. 


\section{2 iRx Patient/Physician/Pharmacist Registration (RPI)}

The registration interface (Fig. 2) is used to register either a patient, physician, or pharmacist.

To register as a patient, certain personally identifiable characteristics of the patient are captured. Some of these include the full name, gender, phone number, date of birth, and digital photo image. The quality of the photo image must meet certain minimum features, including the facial attributes that are enough to generate facial recognition parameters.

Only registered physicians will be able to review and update a patient's iRx prescriptions. The registration of a physician involves collecting some personal and professional information of the physician such as full name, gender, phone number, date of birth, area of specialization, accreditation, current practice, and digital phone image. In addition to positive verification of the professional information, a physical verification via live video is performed.

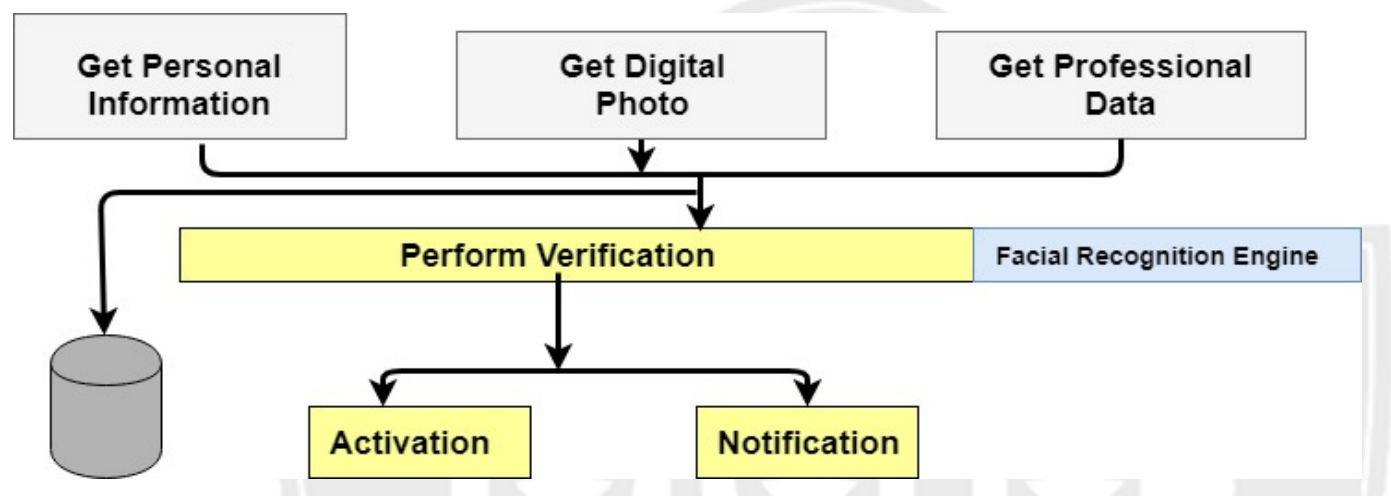

Figure 2: Functional Units for iRx Registration

Only registered pharmacists will be allowed to review and update a patient's iRx prescriptions. During a pharmacist's registration, he/she will be required to provide personal and professional information like that of a physician except for the area of specialization. Just like a physician, a pharmacist's professional information will be verified; and live physical verification will also be conducted.

No one's registration will be deemed completed and activated until all verification activities have been concluded successfully. During any registration, iRx attempts to make sure that no duplicate registration exists. A unique identification number is generated and sent to the registrant. Also, a QRC (Quick Response Code) is generated for every registered patient.

\subsection{Prescriptions Management (PMI)}

iRx prescription management, depicted in Fig. 3, involves either viewing or updating of a patient's prescription history. The owner or registered physician or pharmacist is allowed access to prescription management.

The iRx owner is always allowed access to view or manage their prescriptions (and their dependents) after successful authentication. The system distinguishes between drug information entered by patient or physician/pharmacist. 


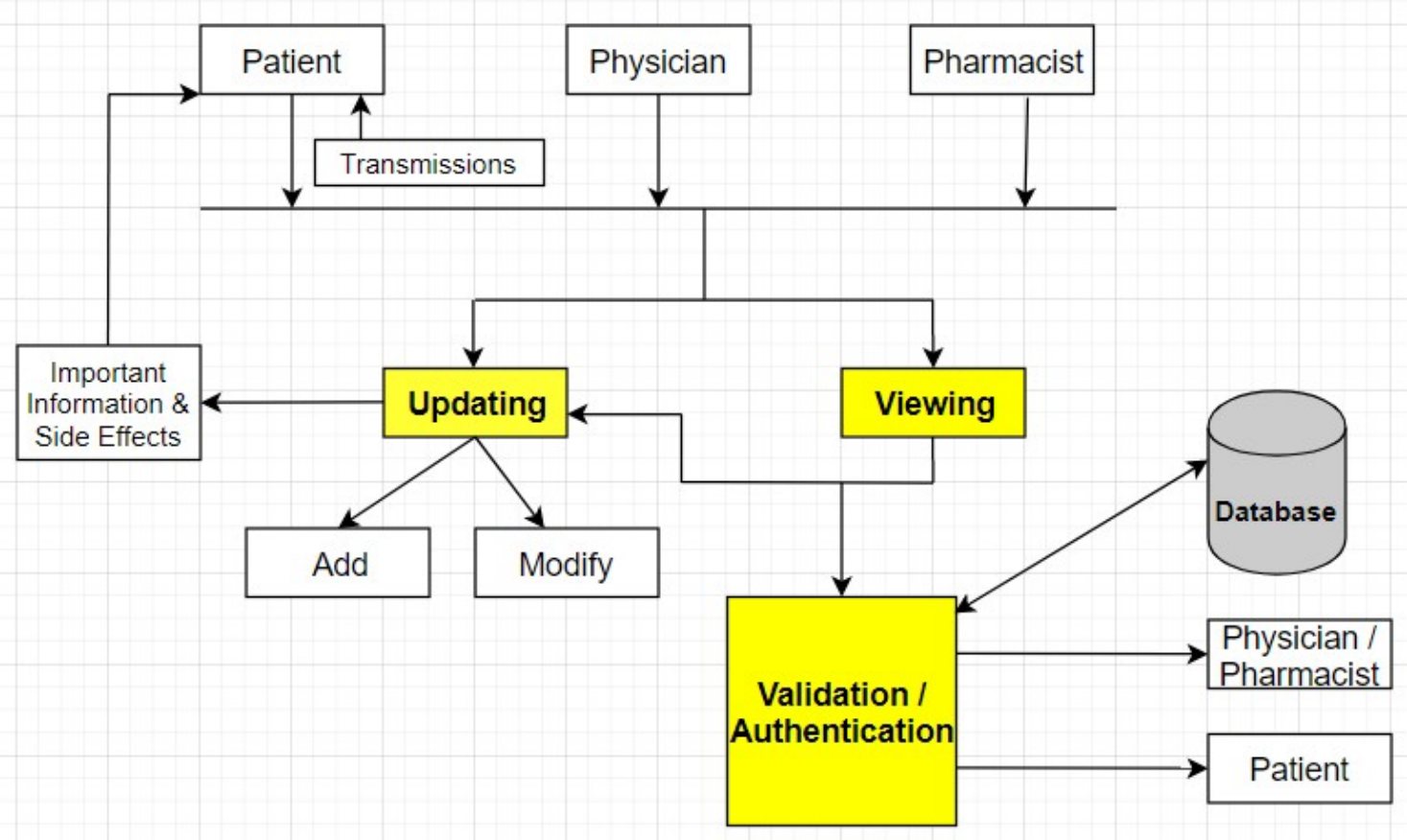

\section{Figure 3: Functional Units for Prescription Management}

Before a physician or pharmacist can access a patient's iRx prescriptions, he/she must be authorized by the patient; and the physician or pharmacist must verify that the holder of the $\mathrm{iRx}$ app is the owner (see Physician/Pharmacist Authorization below). After successful verification and authorization, the requester can then view the necessary information.

In the case of a pharmacist, modifications can be made on the patient's prescription records. When creating a new prescription in $\mathrm{iRx}$, pertinent pieces of information about the medication are stored; these include name, frequency, date filled, expiration date, refill requirements, and prescribing Physician. However, to save the updated or modified data, the patient will be required to review and accept the update/modifications on the patient's mobile device.

As part of the management feature, iRx provides the ability for a patient to electronically transmit selected prescriptions to any participating pharmacy.

\subsubsection{Drug Description and Side Effects}

Some of the most useful information when dispensing medications are about the uses, side effects, and drug interactions associated with the medication. For each medication, IRx provides information for the patient about the its uses; the various side effects; and the interactions with other medications that the patient is currently using. IRx also provides other necessary actions in cases of abuse or emergencies; optimal storage, and helpful notes. These pieces of information will always be accessible to the patient.

\subsubsection{Physician/Pharmacist Authorization}


When a physician or pharmacist needs to view or update a patent's prescription history, he/she must scan the secure QRC that represents the patient's mobile identification. When the patient is not co-located with the requester, the iRx owner ID number can be used instead of the QRC. Authorization is granted by a patient to a co-located requester by displaying the associated QRC of the patient.

\subsection{Prescription Interaction Verification (PIV)}

The Prescription Interaction Interface (PIV), shown in Fig. 4, enables either the patient, physician, or pharmacist to check whether a given drug has any adverse interaction with any of the current active drugs that the patient is taking. To perform this verification by a patient, he/she provides the $i R x$ authentication information, enters or selects the target drug name, and submits the request. The verification result is displayed for the patient.

For example, to perform this verification by either a physician or pharmacist, he/she scans the patient's iRx ID (QRC) from the patient's device; enters or selects the drug name; and submits the request.

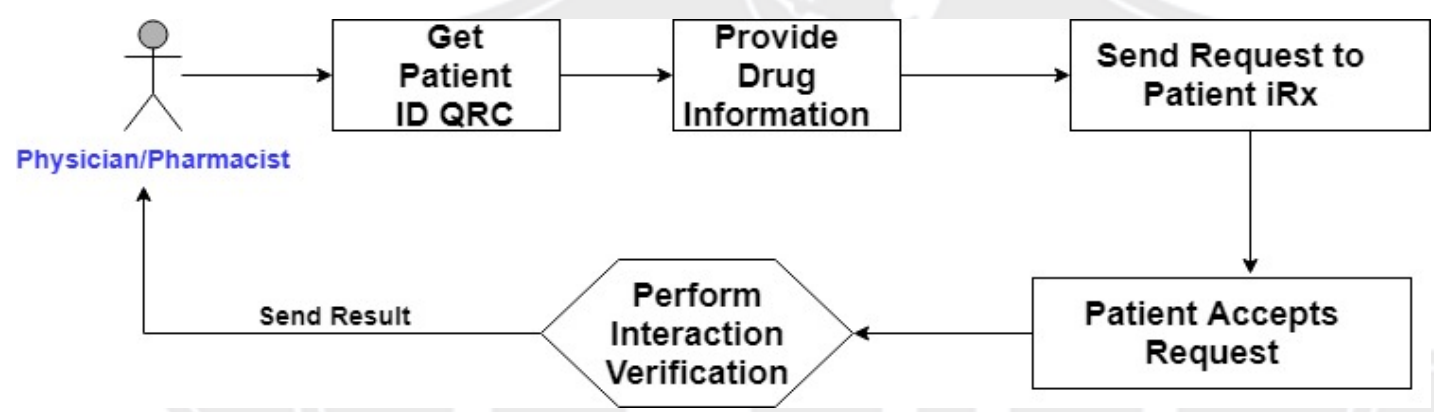

Figure 4: Functional Units for Drugs Interaction Verification

\subsection{Patient Verification Interface (PVI)}

One of the critical elements when accessing a patient's medical information by others is how to guarantee or enforce privacy and security of the applicable information. iRx provides a verification interface that prevents anyone access to a patient's associated medical information without authorization by the patient. When a physician or pharmacist needs to either view or update a patient's medical information, an authorization must be granted by the patient before the request can be successfully performed. This authorization can be performed either when the patient is colocated with or remote from the requester. For example, when the patient is co-located with the requester, the authorization is granted by the patient by displaying the associated QRC for the requester to scan.

\section{IMPLEMENTATION STATUS}

A proof-of-concept prototype of iRx has been developed and tested on the Android mobile platform. It comprises a front-end mobile application and a back-end web server and database. All the key features and functionalities of the system were included in the prototype such as creating 
and updating a physician or patient; creating, updating, and viewing medical records; and physician and patient authorization controls. To download and install on your Android device, go to https://nwosu.net/irx/irx.apk. To download the associated user manual, use https://nwosu.net/irx/manual.pdf.

\section{CONCLUSIONS}

The premise of this paper is to explore for an effective solution to infrastructural problems that effect healthcare delivery, especially as they relate to drugs/prescriptions delivery and management, in communities in under-developed and developing countries. Evidence has shown that these infrastructural problems will persist in those communities for a long time. As a result, solutions that circumvent or do not depend on those infrastructures are needed. iRx is one of such solutions because of its mobile-based platform. In $\mathrm{iRx}$, both providers (physicians and pharmacists) and patients can ubiquitously access a patient's relevant medical information. This system protects a patient's privacy because the patient controls who can and when their medical information is accessible. With the ability to access a patient's medical information, this enables the provider to make sound medical decisions, thereby, drastically reducing the potential for errors that might have resulted in adverse or tragic conditions. A by-product of this system is the possible elimination of one of the prevalent problems in the target communities - the existence of fake or quack physicians and pharmacists.

\section{REFERNCES}

da Cruz, H. L., da Cruz Mota, F. K., Araujo, L. U., Bodevan, E. C., Seixas, S. R. S., \& Santos, D. F. (2017). "The utility of the records medical: factors associated with the medication errors in chronic disease," Revista Latino-Americana de Enfermagem, vol. 25, 2017.

Robertson, J., DeHart, D., Tolle, K., \& Heckerman, D. (2012). "Healthcare Delivery in Developing Countries: Challenges and Potential Solutions," Health and Wellbeing, https://pdfs.semanticscholar.org/bcb6/ce53485b9b2d35b6f4bc6f7280614f4b8682.pdf.

WHO (World Health Organization) (2016) "Medical Errors: Technical Series on Safer Primary Care," ISBN 978-92-4-151164-3, 2016.

Ameh, Y., (2015). “The Challenges of Healthcare Delivery in Developing Countries," BMJ 2005; 330-684, April 2005.

Peters, D. H., Garg, A., Bloom, G., Walker, D. G., Brieger, W. R., \& Rahman, M. H. (2008). "Poverty and Access to Health Care in Developing Countries," Annals of the New York Academy of Science, vol. 1136, Issue 1, pp. 161-171, June 2008.

Mills, A. (2014). "Health Care Systems in Low- and Middle-Income Countries," New England Journal of Medicine, Vol. 370, pp.552-557, Feb. 6, 2014.

Peters, D. H., El-Saharty, S., Siadat, B., Janovsky, K., \& Vujicic, M. (2011). "Improving Health Service Delivery in Developing Countries: From Evidence to Action," Google Books, 2009.

Epstein, M. J., \& Bing, E. G. (2011). "Delivering Health Care to the Global Poor: Solving the Accessibility Problem," Innovations, vol, 6, no. 2, pp. 117-141, 2011.

Bangdiwala, S. I., Fonn, S., Okoye, O., \& Tollman, S. (2010). "Workforce Resources for Health in Developing Countries," Public Health Reviews, Vol. 32, No 1, 296-318, 2010. 
Frost, L. J., \& Reich, M. R. (2009). "Creating Access to Health Technologies in Poor Countries," Health Affairs, vol. 28, NO. 4: Delivering on Global Health, July/Aug. 2009.

American Public Health Association, (2008). "Strengthening Health Systems in Developing Countries," American Public Health Association, Policy Number:20089, Oct. 2008.

Betancur, P. L. (2013). "Nursing and Public Health in Developing Countries," Current Topics in Public Health Alfonso J. Rodriguez-Morales, IntechOpen, DOI: 10.5772/53452, May 2013. Available from: https://www.intechopen.com/books/current-topics-in-publichealth/nursing-and-public-health-in-developing-countries

Strasser, R., Kam, S. M., \& Regaladol, S. M. (2016). "Rural Health Care Access and Policy in Developing Countries," Annual Rev. Pub. health, vol. 36, pp. 395-412, 2016.

Anyika, E. N. (2014). "Challenges of implementing sustainable health care delivery in Nigeria under environmental uncertainty," Journal of Hospital Administration, vol. 3, No. 6, 2014.

United Nations, (2008). International Standard Industrial Classification of All Economic Activities, Rev.3. New York, 2008.

Tam, V. C., Knowles, S. R., Cornish, P. L., Fine, N. I., Marchesano, R., \& Etchells, E.E. (2005). "Frequency, type and clinical importance of medication history errors at admission to hospital: a systematic review", Can Med Assoc J. 2005;173(5):510-515. 